\title{
DIAGNÓSTICOS DE ENFERMAGEM DA CIPE® PARA VÍTIMAS DE ACIDENTE VASCULAR ENCEFÁLICO ISQUÊMICO
}

Natana de Morais Ramos ${ }^{1}$ Jacira dos Santos Oliveira ${ }^{1}$ Maria Naiane Rolim Nascimento ${ }^{2}$ Célida Juliana de Oliveira ${ }^{2}$ Maria Miriam Lima da Nóbrega ${ }^{1}$ Nuno Damácio de Carvalho Félix ${ }^{3}$ https://orcid.org/0000-0002-6194-8757 https://orcid.org/0000-0002-3863-3917 https://orcid.org/0000-0001-9115-1485 https://orcid.org/0000-0002-8900-6833 https://orcid.org/0000-0002-6431-0708 https://orcid.org/0000-0002-0102-3023

Objetivo: Elaborar enunciados de diagnósticos de enfermagem da Classificação Internacional para a Prática de Enfermagem, com vistas ao cuidado intensivo às vítimas de acidente vascular encefálico isquêmico, à luz da Teoria da Adaptação de Roy. Método: Pesquisa metodológica, realizada em três etapas: construção dos enunciados diagnósticos de enfermagem da Classificação, na versão 2017; elaboração das definições operacionais dos enunciados; e validação de conteúdo por especialistas. Resultados: Construíram-se 60 diagnósticos de enfermagem com respectivas definições operacionais. Destes, validaram-se 48 enunciados, com predomínio dos diagnósticos referentes ao modo adaptativo fisiológico. Conclusão: Os diagnósticos de enfermagem validados para cuidado com vítimas de acidente vascular encefálico isquêmico apresentaram potencialidade para proporcionar adaptação do indivíduo e fortalecer o registro das necessidades identificadas por enfermeiros.

Descritores: Terminologia Padronizada em Enfermagem; Diagnóstico de Enfermagem; Acidente Vascular Cerebral; Isquemia Encefálica; Adaptação.

\section{NURSING DIAGNOSTICS FROM ICNP® FOR STROKE VICTIMS}

Objective: To elaborate nursing diagnosis statements of the International Classification for Nursing Practice for the intensive care of victims of ischemic stroke, in light of Roy's Theory of Adaptation. Method: Methodological research, carried out in three stages: construction of the diagnostic nursing statements of the Classification in the 2017 Version; elaboration of operational definitions of statements; and validation of content by experts. Results: Sixty nursing diagnoses were constructed with respective operational definitions. Of these, 48 statements were validated, predominating the diagnoses referring to the physiological adaptive mode. Conclusion: The validated nursing diagnoses for care of victims of ischemic cerebrovascular accident have the potential to adapt the individual and strengthen the registry of the needs identified by the nurses.

Descriptors: Standardized Nursing Terminology; Nursing Diagnosis; Stroke; Brain Ischemia; Adaptation.

\section{DIAGNÓSTICO DE ENFERMERÍA DE LA CIPE® PARA VÍCTIMAS DE ACCIDENTES VASCULARES ISQUÉMICOS}

Objetivo: Elaborar enunciados de diagnósticos de enfermería de la Clasificación Internacional para la Práctica de Enfermería para el cuidado intensivo a las víctimas de accidente vascular encefálico isquémico, a la luz de la Teoría de la Adaptación de Roy. Método: Investigación metodológica, llevada a cabo en tres etapas: construcción de los enunciados diagnósticos de enfermería de la Clasificación, en la versión 2017; elaboración de las definiciones operativas de los enunciados; y validación de contenido por expertos. Resultados: Se construyeron 60 diagnósticos de enfermería con definiciones operativas. De estos, 48 enunciados fueron validados, predominaron los diagnósticos referentes al modo adaptativo fisiológico. Conclusión: Los diagnósticos de enfermería válidos para la atención a las víctimas de accidente vascular encefálico isquémico presentan potencialidad para proporcionar adaptación del individuo y fortalecer el registro de las necesidades identificadas por enfermeros.

Descriptores: Terminología Normalizada de Enfermería; Diagnóstico de Enfermería; Accidente Cerebrovascular; Isquemia Encefálica; Adaptación.

IUniversidade Federal da Paraíba, PB.

2Universidade Regional do Cariri, CE.

3Universidade Federal do Recôncavo da Bahia, BA.

Autor Correspondente: Fabiola Lopes Rodrigues - Email: fabiolla.lopes02@gmail.com

Recebido: 11/12/2019 - Aceito: 19/03/2020 


\section{INTRODUÇÃO}

A Sistematização da Assistência de Enfermagem (SAE) versa sobre a especificidade profissional que envolve ações dinâmicas e inter-relacionadas para realização do cuidado de Enfermagem, com adoção de método para organização do trabalho profissional. Entre os possiveis métodos, tem-se o Processo de Enfermagem, ferramenta que orienta o cuidado de Enfermagem e a documentação da prática profissional, sendo o enfermeiro responsável pelo planejamento, pela organização, execução e avaliação para melhoria da assistência a pacientes ${ }^{(1-2)}$.

O Processo de Enfermagem é composto por etapas, como o histórico, o diagnóstico, o planejamento, a implementação e a avaliação de Enfermagem. O Diagnóstico de Enfermagem consiste na identificação, por enfermeiros, das necessidades prioritárias que carecem de assistência para obter resultados satisfatórios ${ }^{(1)}$, requer o raciocínio clínico para tomada de decisão quanto às intervenções de enfermagem e à utilização de sistema de classificação para registro da prática profissional. Esses vêm sendo cada vez mais objeto de estudo, devido à complexidade e possibilidade de consolidar a Enfermagem enquanto ciência, devendo apresentar aporte teórico como base para o planejamento, utilizando sistema de classificação para padronizar a linguagem profissional direcionada à determinada prioridade de saúde.

Nessa perspectiva, destaca-se o desenvolvimento dos sistemas de classificação em Enfermagem, dentre estes a Classificação Internacional para a Prática da Enfermagem (CIPE ${ }^{\circledR}$ ), de utilização abrangente, incluindo, na versão 2017 , 4.326 termos distribuídos entre dez conceitos organizadores, 1.915 pré-condenados (relativos a diagnósticos/resultados e intervenções de enfermagem) e 2.401 primitivos $^{(3)}$, auxiliando na documentação da assistência de Enfermagem, de modo sistemático, seguro e de qualidade.

Enquanto instrumental tecnológico de informação, essa classificação funciona como sistema de linguagem padronizada, capaz de fornecer dados que embasam o processo decisório de enfermeiros, possibilitando a elaboração de diagnósticos, resultados e intervenções de enfermagem ${ }^{(4)}$. O uso da CIPE ${ }^{\oplus}$ possibilita raciocínio clínico e visão crítica, colaborando para aperfeiçoamento dos conhecimentos de enfermeiros acerca dessa temática ${ }^{(5)}$

No Brasil, desenvolveram-se estudos direcionados a variadas prioridades de saúde envolvendo a elaboração de Diagnósticos de Enfermagem em situações agudas e crônicas, em diferentes especificidades ${ }^{(6-9)}$. Entretanto, evidenciou-se lacuna no conhecimento quanto à terminologia específica para o cuidado de Enfermagem direcionado às vítimas de Acidente Vascular Encefálico (AVE), em especial, oriundo de processo isquêmico.
O AVE é considerado uma das principais causas de morte em escala nacional e internacional, devido ao comprometimento neurológico focal ou global, que incide, subitamente, de provável origem vascular e ocasiona morte ou distúrbios funcionais, a exemplo de hemiplegia, afasia, cegueira, alterações táteis-proprioceptivas, mentais e cognitivas. Essas sequelas acarretam incapacidade total ou parcial da pessoa, gerando grandes implicações na qualidade de vida e elevados gastos financeiros para o sistema de saúde e impactos no orçamento familiar do sujeito doente. Os sintomas incluem ampla variedade de déficits neurológicos, os quais dependem da localização da injúria, do tamanho da área que está com a perfusão insuficiente e da quantidade do fluxo sanguíneo colateral ${ }^{(10)}$

O AVE pode ser classificado como hemorrágico ou isquêmico, sendo a última mais frequente e de prognóstico clínico melhor, caracterizando-se pela cessação do fluxo sanguíneo (obstrução arterial por trombos ou êmbolos), em determinada área do encéfalo, responsável pelas principais causas de internações, resultando em algum tipo de deficiência, seja parcial ou completa, dificultando o retorno da pessoa às atividades diárias e gerando impacto na vida pessoal, familiar e social, transformando cotidianos ${ }^{(11)}$

Quanto ao AVE Isquêmico (AVEI), este é uma prioridade de saúde e insere-se no contexto do cuidado de Enfermagem, com a demanda de identificar as necessidades humanas, por meio dos Diagnósticos de Enfermagem, e a implementação de cuidados intensivos, norteados pela Teoria de Enfermagem ${ }^{12}$ que favorece a promoção da adaptação à condição de saúde e as limitações advindas. Logo, objetivou-se elaborar enunciados de Diagnósticos de Enfermagem, da Classificação Internacional para a Prática de Enfermagem, com vistas ao cuidado intensivo às vítimas de acidente vascular encefálico isquêmico, à luz da Teoria da Adaptação de Roy.

\section{MÉTODO}

\section{Tipo de estudo}

Pesquisa metodológica.

\section{Participantes do estudo}

Enfermeiros especialistas, identificados pela análise na Plataforma Lattes, devendo estes obter a pontuação mínima de cinco pontos e preencher adequadamente os seguintes critérios: mestrado em Enfermagem (quatro pontos); mestrado em Enfermagem, com dissertação relevante para cuidados intensivos (um ponto); pesquisas (com publicações) na área de Diagnósticos de Enfermagem e/ou cuidados intensivos (dois pontos); artigo publicado em periódicos de referên- 
cia para diagnósticos e/ou cuidados intensivos (dois pontos); doutorado voltado para diagnósticos e/ou cuidados intensivos (dois pontos); prática clínica de, pelo menos, um ano de duração na unidade de terapia intensiva (um ponto); e especialização com comprovada prática clínica ou residência em unidade de terapia intensiva (dois pontos). Identificaram-se 55 especialistas, os quais foram convidados a participar via e-mail. Do total, 15 especialistas aceitaram participar do estudo.

\section{Local do estudo}

Estudo realizado com enfermeiros especialistas de vários estados do Brasil.

\section{Coleta dos dados}

Realizou-se de agosto a dezembro de 2018. Os dados foram coletados em três etapas: 1) construção dos enunciados diagnósticos de enfermagem da CIPE $^{\circledR}$; 2) elaboração das definições operacionais dos Diagnósticos de Enfermagem; e 3) validação dos Diagnósticos de Enfermagem.

Na primeira etapa, os enunciados foram construidos com a consultoria de dois enfermeiros atuantes nos cuidados envolvendo vítimas de AVEI, selecionados pelos critérios: possuir titulação mínima de mestre em Enfermagem e atuar em serviços com a SAE implementada.

Utilizaram-se dos termos constantes na CIPE $^{\circledR}$ versão $2017^{13}$, com base no modelo dos Sete Eixos da CIPE ${ }^{\circledR}$, seguindo as recomendações do International Council of Nurses (ICN) e da ISO 18.104:2014 ${ }^{14}$ que trata da integração de um modelo de terminologia de referência para Enfermagem, em que os Diagnósticos de Enfermagem devem ser elaborados por meio da utilização de um termo do Eixo Foco e um termo do Eixo Julgamento ou um achado clínico, podendo, ainda, incluir termos adicionais, se necessário, de outros eixos. Demandou-se a inclusão de termos especificadores em alguns enunciados para vinculá-los diretamente com os fenômenos identificados nas vítimas de AVEI.

Em seguida, procedeu-se à construção das definições operacionais dos diagnósticos, segunda etapa. Seguiram-se as seguintes etapas para elaboração das definições operacionais para os enunciados dos Diagnósticos de Enfermagem: desenvolvimento de definição preliminar; mapeamento do significado das definições dos conceitos; e afirmação da definição operacional(15). Estas fases foram desenvolvidas pelos pesquisadores responsáveis pelo estudo e avaliadas pelos consultores anteriormente referidos.

Para fase de definição preliminar, desenvolveu-se a significação teórica, incluindo ideias-chave e sinônimos, advindos da observação, experiência clínica e/ou literatura.
Esse momento foi fundamental para indicar o objetivo dos conceitos que foram definidos operacionalmente ${ }^{(15)}$. Para o mapeamento das definições, consultaram-se livros, dicionários de termos de Enfermagem e saúde e publicações científicas relacionadas ao cuidado de Enfermagem às vítimas de AVEI. Em seguida, realizou-se a organização das definições dos conceitos relacionados aos Diagnósticos de Enfermagem

A última etapa do estudo versou sobre a validação dos Diagnósticos de Enfermagem. Disponibilizou-se formulário construido no Google Drive Forms ${ }^{\circledR}$, por 30 dias consecutivos para os especialistas participantes do estudo. $\bigcirc$ formulário foi composto pelos Diagnósticos de Enfermagem e pelas respectivas definições, construídas nas etapas anteriores, permitindo que os especialistas avaliassem os itens de forma dicotômica, "concordo" ou "não concordo", utilizando-se do Índice de Validade de Conteúdo (IVC) igual ou superior a 0,80, recomendado por autores no desenvolvimento de outro estudo(16) com o mesmo sistema de classificação. No entanto, ao selecionar "não concordo", os especialistas foram orientados a fornecer sugestões para contribuir com o aprimoramento do estudo.

\section{Procedimento de análise de dados}

Organizaram-se os Diagnósticos de Enfermagem de acordo com os modos adaptativos, devido à inserção destes nas definições propostas pelo referencial teórico, os quais se identificaram de acordo com o código inserido na versão da Classificação. Realizou-se a exportação dos dados da validação para o programa Microsoft Excel for Windows $\circledast$ versão 2016, descritos em tabela e discutidos com a literatura nacional e internacional sobre a temática.

\section{Aspectos éticos}

Este estudo respeitou os preceitos éticos, conforme a Resolução 466/2012 do Conselho Nacional de Saúde, e obteve aprovação do Comitê de Ética em Pesquisa da Universidade Regional do Cariri, sob parecer $n$ ㅇ 1.834.910. Os especialistas atestaram a participação no estudo com a assinatura do Termo de Consentimento Livre e Esclarecido (TCLE).

\section{RESULTADOS}

Elaboraram e definiram-se, operacionalmente, 60 Diagnósticos de Enfermagem da CIPE $^{\circledR}$ versão 2017. Durante o processo de análise pelos especialistas, 48 enunciados foram validados, prevalecendo os classificados no modo Fisiológico (77,1\%), seguidos respectivamente, pelo Autoconceito $(8,3 \%)$, Interdependência $(8,3 \%)$ e Desempenho de papel (6,3\%) (Tabela 1$)$. 
Tabela 1 - Diagnósticos de Enfermagem validados para vítimas de acidente vascular encefálico isquêmico, de acordo com os modos adaptativos de Roy. Brasil, 2018. 


\begin{tabular}{|c|c|c|}
\hline \multirow{4}{*}{ AUTOCONCEITO } & Ansiedade (10000477) & 1,00 \\
\hline & Baixa Autoestima (10029507) & 1,00 \\
\hline & Medo de Representar uma Carga para os Outros (10041671) & 0,87 \\
\hline & Agitação (10025705) & 0,80 \\
\hline \multirow{3}{*}{$\begin{array}{l}\text { DESEMPENHO } \\
\text { DE PAPEL }\end{array}$} & Adaptação, Prejudicada (10022027) & 1,00 \\
\hline & Déficit de Autocuidado (10023410) & 0,87 \\
\hline & Risco de Enfrentamento Familiar, Prejudicado (10032364) & 0,87 \\
\hline \multirow{4}{*}{ INTERDEPENDÊNCIA } & Enfrentamento Familiar, Prejudicado (10034770) & 0,94 \\
\hline & Capacidade de Executar Higiene Oral (ou Bucal), Prejudicada (10029645) & 0,80 \\
\hline & Capacidade para Executar a Higiene Corporal, Prejudicada (10000987) & 0,80 \\
\hline & Alimentação, por si próprio, Prejudicada (10000973) & 0,80 \\
\hline
\end{tabular}

Dentre os Diagnósticos de Enfermagem validados pelos especialistas, 38 foram negativos (79,2\%) e 10 de risco (21,8\%), não contemplando enunciados positivos. Aplicaram-se os termos especificadores "prejudicado", "diminuído" e "cerebral", com finalidade de relacionar diretamente os diagnósticos de enfermagem à prioridade de saúde do estudo.

\section{DISCUSSÃO}

No contexto de Cuidado de Enfermagem direcionado à vítima de AVEI, destaca-se a demanda de aporte teórico, para que se possa estruturar o cuidado efetivo e favoreça a interação entre enfermeiro-paciente-ambiente, na produção de estímulos e respostas ${ }^{c}$, que auxiliem vítimas durante o processo de adaptação ou restabelecimento do processo saúde-doença vivenciado.

Alinhada a tal perspectiva clínica, soma-se a potencial contribuição de enfermeiros, na identificação das necessidades e implementação do cuidado, por meio do Sistema de Classificação em Enfermagem, com foco no cuidado integral, com potencialidade para o registro e a inserção dos indicadores de Enfermagem nos sistemas de informação em saúde. Assim, os enunciados de Diagnósticos de Enfermagem, elaborados e validados neste estudo, colaboram com o cuidado clínico direcionado às vítimas de AVEI, com foco na prática profissional, nos serviços de saúde, com a utilização dos modos adaptativos de Roy.

Neste estudo, o modo fisiológico contemplou a maior quantidade de enunciados para prioridade de saúde e convergiu com estudo(17) que utilizou a taxonomia da NANDA-I, Inc., cujos Diagnósticos de Enfermagem mais prevalentes nessa população foram: Comunicação prejudicada, Deglutição prejudicada, Risco de aspiração, Mobilidade física prejudicada, Reflexo motor diminuído, Risco de queda. Esses enunciados, considerados válidos no presente estudo, demonstram a interseção entre as classificações, se considerados os dois sistemas, a NANDA-I, Inc., e a CIPE ${ }^{\circledR}$.

Especificamente, quanto ao enunciado Comunicação, Prejudicada, 37,5\% das vítimas de AVE apresentaram esse problema ${ }^{(18)}$. Apesar de o estudo não apresentar dados específicos para o tipo isquêmico, o que mais acomete a população, destaca-se a ocorrência de prejuízos na comunicação verbal. $\bigcirc$ enfermeiro utiliza a comunicação para identificar as necessidades de cuidado, assim como desenvolve estratégias alternativas, de forma não verbal, para possibilitá-la. Outra dificuldade comum nessa população relaciona-se ao Diagnóstico de Enfermagem Disfasia, cuja literatura aponta que $86,7 \%$ das vítimas apresentaram dificuldade para formar palavras ou sentenças ${ }^{(18)}$, corroborando com os especialistas deste estudo.

Em relação à Deglutição, Prejudicada, tem-se a necessidade de identificação e intervenção mais precoce possivel, pois está associada, muitas vezes, a várias complicações, como a desidratação, a perda de peso, a má nutrição, o aumento das secreções orais que podem conduzir à obstrução das vias respiratórias ${ }^{(19)}$, elevando o Risco de Aspiração.

Estudo de validação do Diagnóstico de Enfermagem Risco para Aspiração, da NANDA-I, Inc., identificou que fatores de risco, como a deglutição e mobilidade física prejudicadas, são considerados bons preditores para o fenômeno ${ }^{(20)}$. Nesse estudo, elaboraram-se dois enunciados relacionados aos 
preditores referidos, Deglutição, Prejudicada e Mobilidade, Prejudicada, inferindo-se a correlação entre os sistemas de classificação e a importância da identificação precoce para evitar tais fenômenos nas vítimas com AVEI.

O enunciado Mobilidade, Prejudicada relaciona-se com o Risco de Quedas, conforme observado em outro estudo(21). Elaboraram-se enunciados relacionados a alterações de mobilidade, como Paresia. Fenômenos como esses podem apresentar mecanismos de desalinhamento corporal, movimentação incorreta, imobilidade, manuseio e posicionamento inadequado, que podem ocasionar lesões por pressão. Essas sequelas implicam algum grau de dependência e cerca de 30 a $40 \%$ dos sobreviventes são impedidos de voltarem ao trabalho, requerendo auxilio no desempenho das atividades cotidianas básicas, necessitando da ajuda de outras pessoas para atos como a promoção da própria higiene e a realização das necessidades fisiológicas básicas ${ }^{(22)}$.

As alterações na motricidade, no sistema nervoso, deficiência na oxigenação e perfusão cerebral também contribuem para o desenvolvimento de certo grau de dependência dos pacientes, em que, muitas vezes, encontram-se restritos ao leito e impossibilitados para realização de cuidados básicos, o que os tornam susceptíveis à perda da continuidade da pele, devido ao tempo de permanência hospitalizado e às fragilidades que o AVEI causa(23).

Desse modo, enfermeiros devem atentar para tais necessidades e não negligenciar possíveis Diagnósticos de Enfermagem relacionados à integridade da pele, como Risco de Integridade da Pele Prejudicada, Risco de Úlcera por Pressão e Úlcera por Pressão, com potencialidade de ocorrência na assistência às vítimas de AVEI, como relatado pelos especialistas envolvidos neste estudo.

Salienta-se que, neste estudo, houve ampla sugestão dos especialistas para substituição do termo úlcera por lesão, nos Diagnósticos de Enfermagem referidos, devido à modificação conceitual recomendada pelo National Pressure Ulcer Adusory Panel (NPUAP)(24). No entanto, a CIPE ${ }^{\circledR}$ 2017, ainda, utiliza o termo desatualizado, em que se tem a necessidade de discutir e atualizar os conceitos relevantes e adequados para o cuidado de Enfermagem. Destaca-se a elevada incidência dessas lesões em paciente críticos e a multicausalidade dos fatores de risco para o desenvolvimento, para os quais devem ser implementadas estratégias de prevenção, gerenciamento e gestão do cuidado ${ }^{(25)}$.

Para o modo Autoconceito, onstruíram-se Diagnósticos de Enfermagem relacionados ao contexto psicossocial, considerando que as vítimas de AVEI vivenciam alterações psicológicas e emocionais, como ansiedade e depressão(26). Estudo(27) identificou o Diagnóstico de Enfermagem Baixa Autoestima em um caso clínico no contexto. A baixa autoestima pode ocasionar complicações psiquiátricas, sendo a depressão a mais frequente, legitimado por estudo outro desenvolvido ${ }^{28}$. Aponta-se a relevância da atuação de enfermeiros, no tocante aos aspectos psíquicos, uma vez que estes costumam ser permanecidos em segundo plano. Esses profissionais, juntamente com os familiares, devem promover a autoestima dos pacientes, estimulando a participação destes na realização de cuidados básicos ou atividades para desenvolvimento de habilidades, a fim de favorecer adaptação às novas condições clínicas de saúde.

A presença de sequelas gera dependência durante realização das atividades de vida diária por parte dos pacientes, em que $66,18 \%$ das vítimas apresentam dependência entre moderada e severa ${ }^{(29)}$. Esses dados estão relacionados ao modo Desempenho de papel, em que as vítimas de AVEI podem transitar da autonomia para dependência de forma abrupta, o que dificulta o processo de adaptação do paciente, demandando cuidados de Enfermagem para realização dos cuidados básicos.

No modo Interdependência, os enunciados validados destacaram a importância do apoio familiar para esses pacientes, pois não são raros os casos em que a pessoa permanece totalmente dependente psíquica e fisicamente. O período de hospitalização é o principal momento para os familiares iniciarem o aprendizado quanto aos cuidados necessários envolvendo o momento após AVEI, inclusive a higiene oral que tanto é relegada a segundo plano. O processo de recuperação do paciente, de forma geral, é lento e depende da assistência de equipe multiprofissional(30)

Pesquisa mostra que as práticas de higiene oral, quando realizadas de forma correta, são bastante benéficas aos pacientes críticos, pois estas reduzem a presença de bactérias que se alojam na cavidade oral(31) que podem se deslocar para outros locais e ocasionar doenças oportunistas, o que complicaria o quadro clínico do paciente. Logo, enfermeiros devem identificar as necessidades e respostas humanas das vítimas de AVEI, considerando os modos adaptativos, as variações oriundas da individualidade das pessoas e a interação familiar e social destas.

Diante dessas necessidades, cabe aos profissionais fornecerem informações à pessoa e aos familiares que thes permitam readaptar-se à nova situação de saúde ${ }^{(29)}$. A necessidade de cuidado direto e a incapacidade de realizar atividades simples podem ser dispendiosos para os familiares. Perante tais mudanças, a pessoa doente e família têm que aprender a viver com as novas condições e conhecer estratégias que permitam readaptar-se, para que o sentimento de bem-estar, motivação, coragem, fé possam ser preservados(31). 


\section{Limitações do estudo}

Ao considerar o processo de pesquisa, teve-se como limitação o fato de que os termos utilizados para elaborar os enunciados Diagnósticos de Enfermagem não foram extraídos da prática clínica de enfermeiros com pessoas vítimas de AVEI, devido à dificuldade em acessar esses serviços especializados. No entanto, tal restrição foi amenizada, considerando que se construíram os Diagnósticos de Enfermagem utilizando-se dos termos constantes em um Sistema de Classificação de Enfermagem reconhecido internacionalmente e, posteriormente, validados por especialistas na área.

\section{Contribuições para a prática}

Apresenta-se o fornecimento de subsídio terminológico para facilitar o registro do cuidado de Enfermagem à vítima de AVEI, nos variados serviços de saúde, previamente avaliados por especialistas, favorecendo a geração de indicadores de cuidado mensuráveis para fomento da relevância da Enfermagem e respectivo impacto na saúde da população.

\section{CONCLUSÃO}

Elaboram-se enunciados Diagnósticos de Enfermagem para o cuidado às vítimas de AVEI, à luz da Teoria da Adaptação de Roy, em que se ponderaram 48 enunciados válidos pelos especialistas participantes do estudo, predominando o modo adaptativo fisiológico. Enfatiza-se a contribuição para avanço no conhecimento sobre as necessidades de cuidado de Enfermagem, além de promover maior entendimento e consolidação da classificação, o desenvolvimento da terminologia específica e estruturação futura de um subconjunto terminológico da CIPE ${ }^{\circledR}$ para subsidiar o registro do Processo de Enfermagem.

O presente estudo apresenta enunciado que contribui para o conhecimento dos fenômenos de enfermagem potenciais no cuidado à vítima de AVEI, de modo a auxiliar geração de evidências futuras para disciplina e prática clínica, com impacto na constituição de indicadores clínicos de enfermagem para prioridade de saúde eleita. Tem-se linguagem padronizada, baseada em sistema de classificação internacionalmente conhecido, a $\mathrm{CIPE}^{\oplus}$, colaborando, portanto, para o progresso da Prática Avançada de Enfermagem.

\section{REFERÊNCIAS}

1.Conselho Federal de Enfermagem. Resolução n 358, de 15 de outubro de 2009. Dispõe sobre a Sistematização da Assistência de Enfermagem SAE - em ambientes públicos e privados em que ocorre o cuidado profissional de Enfermagem e da outras providencias [Internet]. Brasilia; 2009 [cited 2018 Mar 08]. Available from: http://www.cofen.gov.br/resoluo-cofen-3582009_4384.html

2.Gutiérrez MGR, Morais SCRV. Systematization of nursing care and the formation of professional identity. Rev Bras Enferm [Internet] 2017[cited 2017 Jun 10];70(2):436-41. Available from: http://www.scielo.br/scielo.php?script=sci_arttext\&pid=S0034-71672017000200436

3.Garcia TR, Bartz CC, Coenen AM. CIPE®: uma linguagem padronizada para a prática profissional. In Garcia TR, organizadora. Classificação Internacional para a Prática de Enfermagem (CIPE®): versão 2017. Porto Alegre: Artmed, 2017. Cap. 2, p.24-39.

4.Clares JWB, Guedes MVC, Silva LF, Nóbrega MML, Freitas MC. Subset of nursing diagnoses for the elderly in Primary Health Care. Rev Esc Enferm USP [Internet]. 2016 [cited 2017 Jan 10];50(2):270-6. Available from: http://www.scielo.br/scielo.php?script=sci_ arttext\&pid=S0080-62342016000200272

5.Primo CC, Trevizani CC. Tedesco JC. Leite FMC, Almeida MVS, Lima EFA. Classificação Internacional para a Prática de Enfermagem na assistência pré-natal. Enferm. Foco [Internet]. 2015 [cited 2020 Mar 15]; 6 (1/4):17-23. Available from: http://revista.cofen.gov.br/index.php/enfermagem/ article/view/571/253

6.Silva RS, Pereira A, Nóbrega MML, Mussi FC. Construction and validation of nursing diagnoses for people in palliative care. Rev Latino-Am Enfermagem [Internet]. 2017:25:e2914. Available from: http://www.scielo.br/scielo.php?script=sci_arttext\&pid=S0104-11692017000100362

7.Dantas AMN, Silva KL, Nóbrega MML. Validation of nursing diagnoses, interventions and outcomes in a pediatric clinic. Rev Bras Enferm [Internet] 2018 [cited 2018 Out 15];71(1):80-8. Available from: http://www.scielo.br/scielo.php?script=sci_arttext\&pid=S0034-71672018000100080\&lng=en Enrm=iso\&tlng=pt

8.Souza Neto VL, Silva RAR, Silva CC, Negreiros RV, Rocha CCT, Nóbrega MML. Proposal of nursing care plan in people hospitalized with AIDS. Rev Esc Enferm USP [Internet]. 2017 [cited 2018 Nov 02]:51:e03204. Available from: http://www.scielo.br/scielo.php?script=sci_ arttextEpid=S0080-62342017000100406

9.Félix NDC, Ramos NM, Nascimento MNR, Moreira TMM, Oliveira CJ. Nursing diagnoses from ICNP® for people with metabolic syndrome. Rev Bras Enferm [Internet]. 2018 [cited 2019 Mar 12]:71(Suppl 1):467-74. Available from: http://www.scielo.br/scielo.php?script=sci_arttextEpid=S0034$71672018000700467 \&$ lng=en\&nrm=iso\&tlng=pt

10.Ministério da Saúde (BR). Secretaria de Atenção à Saúde. Departamento de Ações Programáticas Estratégicas. Diretrizes de atenção à reabilitação da pessoa com acidente vascular cerebral / Ministério da Saúde, Secretaria de Atenção à Saúde, Departamento de Ações Programáticas Estratégicas [Internet]. Brasilia, 2013 [cited 2018 Nov 08]. Available from: http://bvsms.saude.gov.br/bvs/publicacoes/diretrizes_ atencao_reabilitacao_acidente_vascular_cerebral.pdf 
11.Rolim CLRC, Martins M. Quality of care for ischemic stroke in the Brazilian Unified National Health System. Cad Saúde Pública [Internet]. 2011 [cited 2019 Mar 20];27(11):2106-16. Available from: http://www.scielo.br/scielo.php?script=sci_arttext\&pid=\$0102-311X2011001100004\&lng=en\&n rm=iso\&tlng=pt

12.Roy C. Andrews HA. The Roy Adaptation Model. 3th. New Jersey: Pearson, 2009

13.International Council of Nurses (ICN). International Classification for Nursing Practice (ICNP@). Genebra: 2018. [Internet] [cited 2018 Jun 24]. Available from: http://www.old.icn.ch/what-we-do/international-classification-for-nursing-practice-icnpr/

14.International Organization for Sandardization. Health Informatics--Categorial structures for representation of Nursing diagnoses and Nursing actions in terminological sistems. (ISO/FDIS 18.104:2014). Genebra: 2014.

15.Lenz ER. Operacionalization Nursing Concepts. In Waltz CF, Strickland OL, Lenz ER. Measurement in nursing and health research. 4. ed. New York (NY): Springer Publishing Company: 2010. 504p.

16.Andrade LT, Garcia TR, Chianca TCM. Diagnósticos e intervenções de enfermagem para o componente sentidos da teoria de Roy, aplicados a adultos em neurorreabilitação. Enferm Foco [Internet]. 2017[cited 2019 Dez 13]; 8(3):45-50. Available from: http://revista.cofen.gov.br/index.php/ enfermagem/article/view/1268/398

17.Lima ACMACC, Silva AL, Guerra DR, Barbosa IV, Bezerra KC, Oriá MOB. Nursing diagnoses in patients with cerebral vascular accident: an integrative review. Rev Bras Enferm [Internet]. 2016 [cited 2018 Mar 25];69(4):738-45. Available from: http://www.scielo.br/scielo.php?script=sci_ arttext\&pid=S0034-71672016000400785\&lng=en\&nrm=iso\&tlng=en

18.Chaves DBR, Costa AGS, Oliveira ARS, Silva VM, Araújo TL, Lopes MVO. Impaired verbal communication - research in the post cerebrovascular accident. Rev Rene [Internet]. 2013 [cited 2019 Mar 20];14(5):877-85. Available from:

19.Oliveira ARS, Costa AGS, Morais HCC, Cavalcante TF, Lopes MVO, Araujo TL. Clinical factors predicting risk for aspiration and respiratory aspiration among patients with Stroke. Rev Latino-Am Enfermagem [Internet]. 2015 [cited 2019 Abr 10]:23(2):216-24. Available from: http://www. periodicos.ufc.br/rene/article/view/3609

20.Cavalcante TF, Araujo TL, Moreira RP, Guedes NG, Lopes MVO, Silva VM. Clinical validation of the nursing diagnosis Risk for Aspiration among patients who experienced a cerebrovascular accident. Rev Latino-Am Enfermagem [Internet]. 2013 [cited 2018 Mar 02];21:250-8. Available from: http://www.scielo.br/scielo.php?script=sci_arttext\&pid=S0104-11692013000700031

21.Correia A, Pimenta C. Virella D. Agreement between instruments for assessing balance after stroke. Saúde $\&$ Tecnologia [Internet]. 2017 [cited 2018 Jun 12]:17:33-8. Available from: https://web.estesl.ipl.pt/ojs/index.php/ST/article/view/1701/1571

22.Lopes Junior JEG, Freitas Junior HA, Figueiredo ADJ, Santana FM. Perfil dos pacientes acometidos por acidente vascular encefálico cadastrados na estratégia de saúde da familia. Rev Fisioter S Fun [Internet]. 2013 [cited 2018 Jan 13];2(1):21-7. Available from: http://www. fisioterapiaesaudefuncional.ufc.br/index.php/fisioterapia/article/view/193

23. Nascimento KG, Chavaglia SRR, Pires PS, Ribeiro SBF, Barbosa MH. Clinical outcomes of ischemic stroke patients after thrombolytic therapy. Acta Paul Enferm [Internet]. 2016 [cited 2018 Jun 22]:29(6):650-7. Available from: http://www.scielo.br/scielo.php?pid=S0103210020160006006508 script=sci_arttext\&tlng=en

24. National Pressure Ulcer Advisory Panel. Pressure Ulcer Stages Revised. [Internet]. Washington, 2016. [cited 2018 Jan 15] Available from: http:// www.npuap.org/about-us/

25.Otto C, Schumacher B, Wiese LPL, Ferro C, Rodrigues RA. Fatores de risco para o desenvolvimento de lesão por pressão em pacientes criticos. Enferm. Foco [Internet]. 2019 [cited 2020 Mar 15]; 10 (1): 07-11. Available from: http://revista.cofen.gov.br/index.php/enfermagem/article/ view/1323/485

26.Loupis YM, Faux SG. Family Conferences in stroke rehabilitation: a literature review. J Stroke Cerebrovasc Dis [Internet]. 2013 [cited 2019 Mar 01]:22(6):883-93. Available from: https://linkinghub.elsevier.com/retrieve/pii/S1052-3057(12)00408-9

27.Brito-Brito PR. Fernandez-Gutierrez DA, Smith HM. Case study: community nursing care plan for a man with functional and psychosocial problems following a stroke. Int J Nurs Knowl [Internet]. 2015 [cited 2018 Jul 25];25(1):62-5. Available from: https://www.ncbi.nlm.nih.gov/ pubmed/25774001

28.Costa TFC, Costa KNFM, Fernandes MGM, Martins PK, Brito SS. Quality of life of caregivers for patients of cerebrovascular accidents: association of (socio-demographic) characteristics and burden. Rev Esc Enferm USP [Internet]. 2015 [cited 2018 Dez 05]:49(2):245-52. Available from: http://www.scielo.br/scielo.php?script=sci_arttextEpid=S0080-62342015000200245

29.Faria ACA, Martins MM, Schoeller SD, Matos LO. Care path of person with stroke: from onset to rehabilitation. Rev Bras Enferm [Internet]. 2017[cited 2018 Mar 06];70(3):495-503. Available from: http://www.scielo.br/scielo.php?script=sci_arttextEpid=\$0034-71672017000300495

30.Guimarães GR, Queiroz APG, Ferreira ACR. Establishment of a oral hygiene protocol in hospitalized patients at the ICU HUSF. Braz $\mathrm{J}$ Periodontol [Internet]. 2017 [cited 2018 Abr 15];27(1):07-10. Available from: http://www.revistasobrape.com.br/arquivos/2017/marco/REVPERIO_ MAR\%C3\%870_2017_PUBL_SITE_PAG-07_A_10\%20-\%2027-03-2017.pdf

31.Araújo JS, Silva SED, Santana ME, Santos LS, Sousa RF, Conceição VM, et al. The parallel side of caring unveiled by caregivers of patients sickened after stroke. R bras ci Saúde [Internet]. 2014 [cited 2016 Fev 12]:18(2):109-14. Available from: https://periodicos.ufpb.br/ojs2/index.php/ rbcs/article/view/14105 